\title{
A xenograft model of macrophage activation syndrome amenable to anti-CD33 and anti-IL-6R treatment
}

\author{
Mark Wunderlich, ${ }^{1}$ Courtney Stockman, ${ }^{1}$ Mahima Devarajan, ${ }^{1}$ Navin Ravishankar, ${ }^{1}$ \\ Christina Sexton, ${ }^{1}$ Ashish R. Kumar, ${ }^{2}$ Benjamin Mizukawa, ${ }^{1}$ and James C. Mulloy
}

'Division of Experimental Hematology and Cancer Biology and 'Division of Bone Marrow Transplantation and Immune Deficiency, Cancer and Blood Disease Institute, Cincinnati Children's Hospital Medical Center (CCHMC), Cincinnati, Ohio, USA.

\begin{abstract}
Transgenic expression of key myelosupportive human cytokines in immune-deficient mice corrects for the lack of cross-species activities of stem cell factor (SCF), IL-3, and GM-CSF. When engrafted with human umbilical cord blood (UCB), these triple-transgenic mice produce $B M$ and spleen grafts with much higher myeloid composition, relative to nontransgenic controls. Shortly after engraftment with UCB, these mice develop a severe, fatal macrophage activation syndrome (MAS) characterized by a progressive drop in rbc numbers, increased reticulocyte counts, decreased rbc half-life, progressive cytopenias, and evidence of chronic inflammation, including elevated human IL-6. The BM becomes strikingly hypocellular, and spleens are significantly enlarged with evidence of extramedullary hematopoiesis and activated macrophages engaged in hemophagocytosis. This manifestation of MAS does not respond to lymphocyte-suppressive therapies such as steroids, i.v. immunoglobulin, or antibody-mediated ablation of human B and T cells, demonstrating a lymphocyte-independent mechanism of action. In contrast, elimination of human myeloid cells using gemtuzumab ozogamicin (anti-CD33) completely reversed the disease. Additionally, the IL-6R antibody tocilizumab delayed progression and prolonged lifespan. This new model of MAS provides an opportunity for investigation of the mechanisms driving this disease and for the testing of directed therapies in a humanized mouse.
\end{abstract}

Conflict of interest: The authors have declared that no conflict of interest exists.

Submitted: April 21, 2016 Accepted: August 16, 2016 Published: September 22, 2016

Reference information: JCI Insight. 2016;1(15):e88181. doi:10.1172/jici.insight.88181

\section{Introduction}

Macrophage activation syndrome (MAS) is a complication in a variety of rheumatoid disorders (1). It is most notably associated with systemic onset juvenile idiopathic arthritis (SoJIA), where studies have found evidence of frequent subclinical episodes that often progress to overt, potentially fatal disease (2-4). It also occurs in Kawasaki disease and systemic lupus erythematosus and sporadically in a myriad of other syndromes (5). MAS is the result of an uncontrolled, hyperactive immune response, often to an identifiable infection but in many cases lacking a known trigger (6). High levels of T cell-produced cytokines such as IFN $\gamma, \mathrm{TNF} \alpha$, and GM-CSF result, activating macrophages and other myeloid cells. These activated macrophages attack cells and tissues directly through phagocytosis and contribute to persistent systemic inflammation via release of IL-1 and IL-6, as well as other proinflammatory cytokines. This unchecked cytokine production leads to a cytokine storm, resulting in widespread organ damage, and is believed to be a key factor in progression of the disease. MAS occurs along a broad spectrum of intensity, with many mild cases likely going undiagnosed while severe cases lead to life-threatening complications.

MAS is frequently classified as a subtype of hemophagocytic lymphohistiocytosis (HLH). Many cases of HLH result from specific mutations, leading to impaired cytotoxicity of T or NK cells. These cases of primary HLH involve defects in the production, processing, or release of perforin by cytotoxic $\mathrm{T}$ and NK cells. Other cases of acquired $\mathrm{HLH}$ are often associated with malignancies or viral infections or other immune-activating processes. The precise mechanisms of secondary HLH are poorly understood. Regardless of the initiating event for these diseases, the final condition is defined by an overactive immunity that leads to chronic inflammation and hemophagocytosis. The existing treatments for all HLH-like conditions are very similar, as well, relying on various immune suppressive regimens of dexamethasone or other steroids, cyclosporine, and etoposide. These therapies are, themselves, toxic 
and add to the morbidity of the already sick patient. Moreover, this approach is likely to be most effective against lymphoid drivers of disease. While the principal pathology in primary/familial HLH is indeed uncontrolled activation of lymphocytes, this is not the case in secondary HLH or MAS. These treatments may thus not be appropriate for MAS (7). Newer therapies such as the IL-6 receptor (IL-6R) inhibitor tocilizumab, the IL-1 receptor antagonist anakinra, and TNF $\alpha$ inhibitors are all being evaluated for efficacy and safety in patients with HLH or MAS (6).

Numerous mouse models now exist that fulfill as few as 0 and as many as all 8 of the HLH diagnostic criteria (8). Many are built upon genetic KO of the key genes identified in primary HLH cases. Several others have been created to model secondary HLH/MAS. All of these existing models require activation of the immune system by a trigger — such as lymphocytic choriomeningitis virus (LCMV) (9), Epstein-Barr virus (EBV) (10), or cytomegalovirus (CMV) (11) — or by stimulation of TLRs (12). These models have been invaluable in the identification of new therapeutic strategies and for exploring the mechanisms behind the disease (13-15). However, there are still cases that lack any identified genetic cause or trigger. Many cases are refractory to available therapies, and others develop in patients already receiving immunosuppressive therapy for other disorders. A lymphocyte-independent, effector-driven model would allow for a more focused examination of the contributions of the monocyte/macrophage arm to MAS/HLH and may result in identification of new therapeutic strategies that may be applicable to these difficult cases.

The NOD/SCID-IL2R $\gamma^{\text {null }}$ (NSG) and NOD/RAG-IL2R $\gamma^{\text {null }}$ (NRG) mouse strains have a complete lack of lymphoid cells and have additional defects in innate immunity. As a result, human cell engraftment in NSG and NRG mice is more efficient and durable, and it includes T cell development that had been lacking in older models, making these strains among the best options for creating a human immune system mouse (16-19). However, these mice allow only limited human myeloid repopulation, which results in ineffective development of functional immunity. In order to address this problem, our lab and others generated mice (here called NSGS and NRGS) with transgenic expression of human stem cell factor (SCF), IL-3, and GM-CSF, circumventing the problem of poor cross reactivity of the corresponding murine cytokines with human receptors. The result is a mouse with superior human myeloid cell engraftment and output (20-24).

Here, we demonstrate the development of MAS in umbilical cord blood-engrafted (UCB-engrafted) NSGS and NRGS mice. Upon engraftment, mice rapidly develop a progressive anemia without the need for exogenous immune stimulation. The mice fulfill many of the diagnostic criteria of secondary HLH/MAS, including pancytopenia, splenomegaly, hemophagocytosis, fever, and increased soluble CD25 (sCD25). This model does not respond to human lymphoid cell ablation; however, mice make full recoveries after treatment with gemtuzumab ozogamicin, indicating a $\mathrm{CD}_{3} 3^{+}$cell is required for the observed phenotypes. Mice with active MAS have increased inflammatory cytokines, including IL-6. Targeting of this pathway with the IL-6R inhibitor, tocilizumab, slowed disease progression and led to an increased lifespan. This effector-driven model should be useful to assess the value of therapies that target activated macrophages that are central to both the inflammation and phagocytic aspects of HLH and MAS. Effector-specific (lymphoid-sparing) treatments would likely also be less immunosuppressive, potentially reducing the morbidity.

\section{Results}

UCB-engrafted NSGS mice develop fatal hemophagocytosis. We found that humanized NSGS mice (hu-NSGS) had a significantly shorter lifespan relative to conditioned, nonhumanized NSGS mice or hu-NSG mice. For example, in one experiment, 5 of 16 hu-NSGS became moribund between 16-20 weeks of engraftment, while 0 of 19 hu-NSG became ill. Gross necropsy revealed pale bones and organs, as well as enlarged

Table 1. UCB engrafted NSCS mice are pancytopenic.

\begin{tabular}{|c|c|c|c|c|c|}
\hline $\begin{array}{l}\text { Mouse } \\
\text { Strain }\end{array}$ & $\begin{array}{l}\text { WBC } \\
(K / \mu l)\end{array}$ & $\begin{array}{l}\text { RBCs } \\
(M / \mu l)\end{array}$ & $\begin{array}{l}\text { Hemoglobin } \\
\text { (g/dl) }\end{array}$ & $\begin{array}{c}\text { Hematocrit } \\
(\%)\end{array}$ & $\begin{array}{c}\text { Platelets } \\
(\mathrm{M} / \mu \mathrm{l})\end{array}$ \\
\hline $\begin{array}{l}\text { NSG (14) } \\
\text { (14 weeks) }\end{array}$ & $2.95 \pm 0.54$ & $8.46 \pm 0.72$ & $13.9 \pm 1.0$ & $52.8 \pm 4.2$ & $987 \pm 156$ \\
\hline $\begin{array}{l}\text { NSGS (25) } \\
\text { (14 weeks) }\end{array}$ & ${ }^{\mathrm{A}} 1.87 \pm 0.65$ & ${ }^{A} 3.92 \pm 0.83$ & $\mathrm{~A} 8.1 \pm 1.5$ & АЗ33.2 \pm 6.0 & A $794 \pm 248$ \\
\hline
\end{tabular}


A

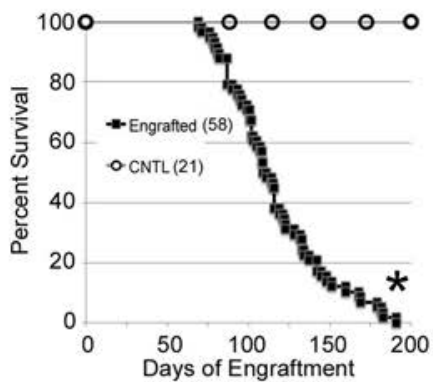

D

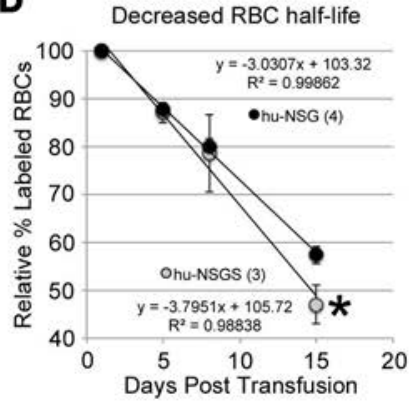

H
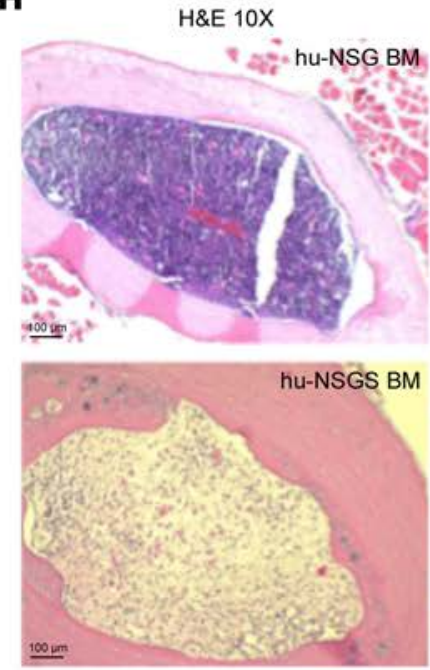

B Development of anemia in hu-NSGS

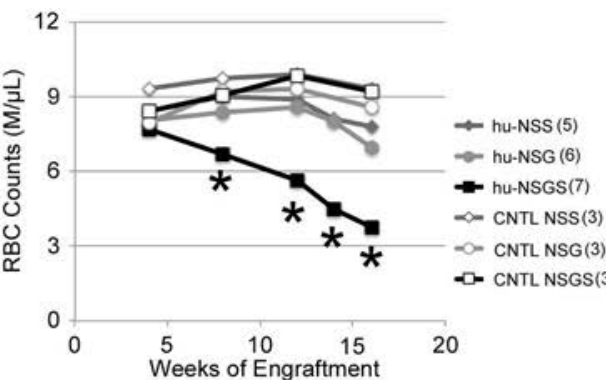

C Increased reticulocytes in hu-NSGS

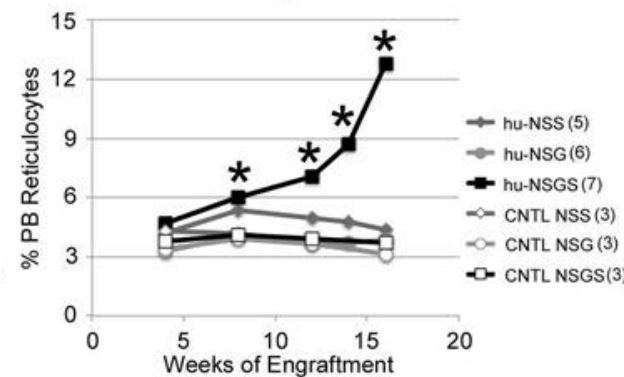

E

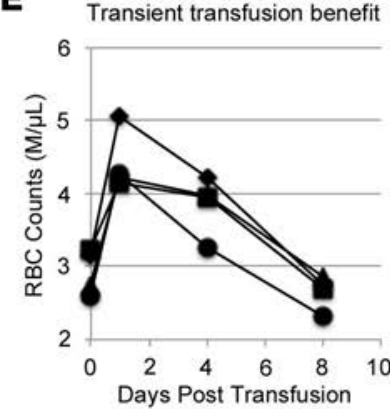

$\mathrm{H} \& \mathrm{E} 40 \mathrm{X}$
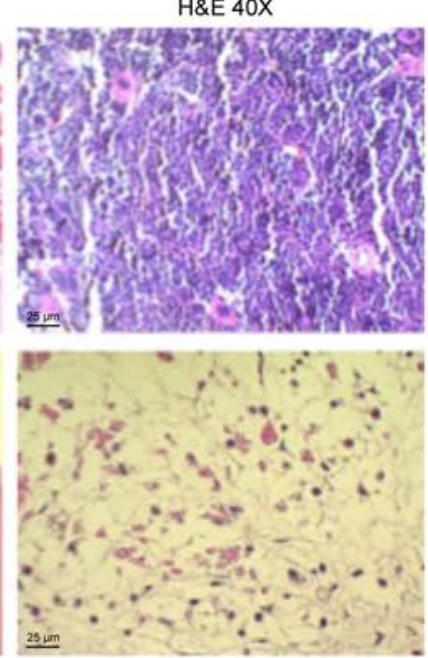

F

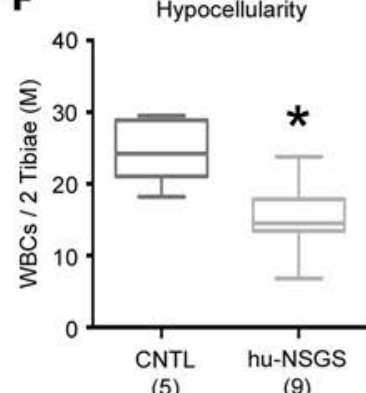

(5)

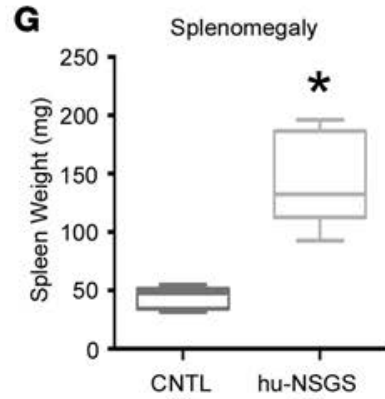

(5) (9)

I

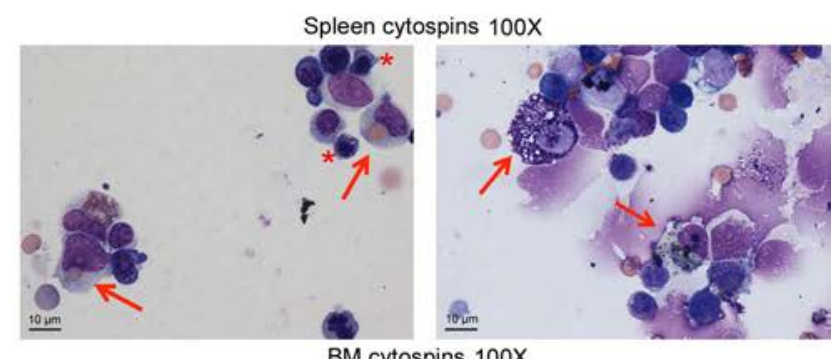

BM cytospins $100 \mathrm{X}$

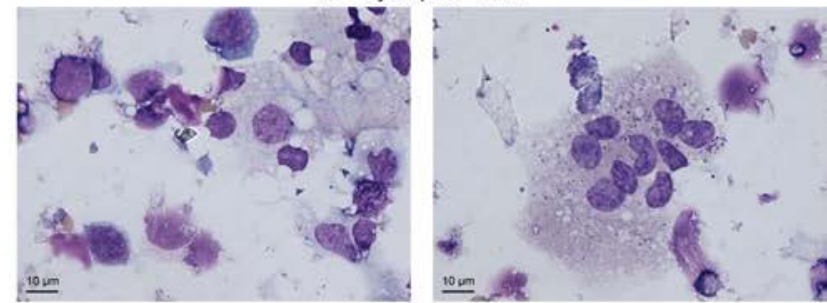

Liver cytospins $40 \mathrm{X}$
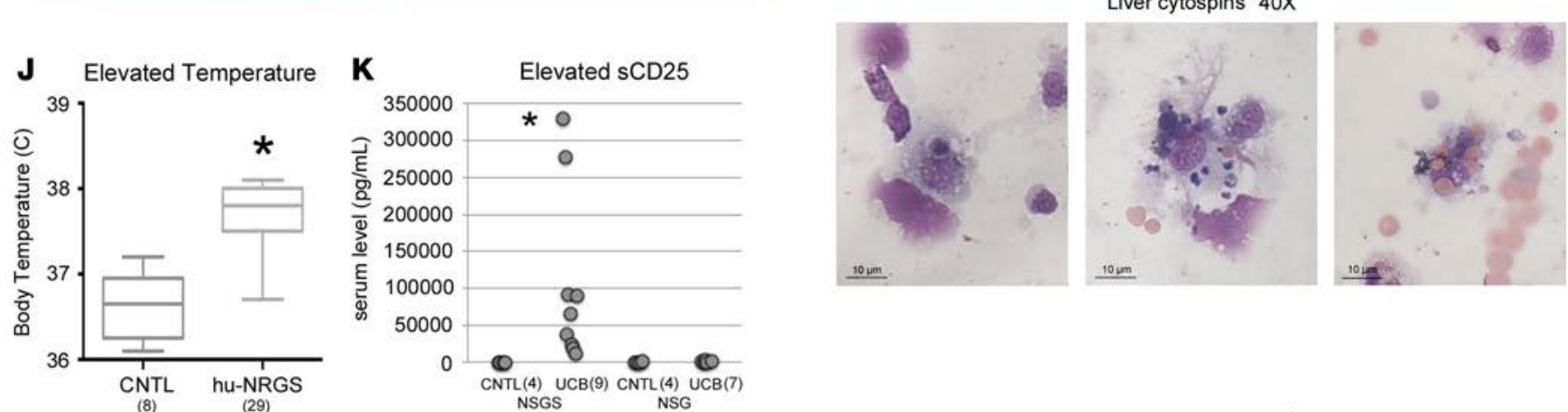

Figure 1. Characterization of MAS in NSGS mice. (A) Survival of UCB-engrafted NSCS mice and nonengrafted control (CNTL) NSCS mice. (B) Serial rbc counts from NSG, NSS, and NSGS mice engrafted with the same UCB and nonengrafted controls. Average result is shown for each group. (C) Peripheral blood samples from B were analyzed for reticulocytes. (D) Persistence of CFSE-labeled rbcs in UCB-engrafted NSC and NSCS mice over time. Small-volume serial bleeds were performed, and CFSE-positive cells were enumerated by flow cytometry. Half-life was determined by solving for the equation of the line of best fit generated by Excel. (E) Serial rbc counts from anemic mice receiving transfusions. Transfusions occurred just after the 
baseline bleed at day 0. (F) Total BM cell counts of nonengrafted CNTL or UCB-engrafted NSCS mice. (G) Spleens from sacrificed animals in F were weighed. (H) H\&E stains of formalin-fixed femurs from engrafted mice. (I) Spleen, BM, and liver samples from engrafted NSCS mice were spun onto slides and stained with Wright-Giemsa. (J) Rectal temperatures were determined for active control nonengrafted mice and UCB-engrafted hu-NRCS mice. (K) Soluble IL2-R $\alpha$ (sCD25) levels in the plasma of engrafted NSG and NSCS mice were determined by ELISA. ${ }^{*} P<0.05$ by Mann-Whitney $U$ test. Panels F, G, and J are box and whisker plots with the bounds of the box indicating the 1st and 3rd quartiles, the line within the box showing the mean, and the whiskers comprising the range of all data points.

spleens in these moribund hu-NSGS. Importantly, there was no evidence of $\mathrm{T}$ cell-mediated graft versus host disease (GVHD) (data not shown). Analysis of a large number of hu-NSGS showed that $100 \%$ of mice died between 10 and 27 weeks of engraftment, while all nonengrafted control mice remained alive and well (Figure 1A). The peripheral blood (PB) of mice at 14 weeks revealed anemia, as indicated by significantly lower rbc counts, hemoglobin, and hematocrit in hu-NSGS relative to hu-NSG (Table 1, $P<0.05$, Mann-Whitney $U$ test). Additionally, the wbc and platelet counts were also significantly lower, indicating a pancytopenia involving all major blood lineages.

To further investigate this finding, we engrafted several strains of mice in parallel with identical UCBs. Serial analysis of PB showed a rapid decline of rbc numbers in hu-NSGS but not in conditioned nonengrafted mice, effectively ruling out long-term effects of exposure to busulfan conditioning (Figure 1B). While hu-NSG and hu-NSS (NSGS with WT IL-2R $\gamma$ ) mice showed some minor trend toward lower rbc counts over time, these were much slower to appear and were not accompanied by the dramatic increase in reticulocyte counts that were consistently observed in hu-NSGS (Figure 1C).

The reticulocytosis suggests that the anemia we observed was due to increased consumption or destruction of erythrocytes. To further investigate the mechanism of the anemia, we labeled rbcs from donor mice with CFSE dye, injected them into hu-NSG and hu-NSGS animals, and monitored their persistence over time. The estimated half-life of rbcs in hu-NSG was 17.6 days, while it was only 14.7 days in hu-NSGS (Figure 1D). In addition, transfusion of whole unlabeled blood provided only a minor, transient benefit to anemic hu-NSGS (Figure 1E). This data suggests that a consumptive process, rather than a production problem, is occurring in hu-NSGS.

Additionally, we found a significantly lower marrow cellularity (Figure 1F) and a marked splenomegaly (Figure 1G) that became more pronounced over time. An example of an advanced case of BM hypocellularity with abundant fatty tissue is shown (Figure $1 \mathrm{H}$ ). Evidence of hemophagocytosis was readily observed in hu-NSGS spleens, along with abundant nucleated rbc precursors (asterisks) and hemosiderin-laden macrophages (arrows, Figure 1I, top row). The BM showed dominant myeloid engraftment (middle left) with frequent giant cells (middle right) generated from macrophage fusion, likely indicating chronic inflammation. Hemophagocytosis was also readily found in the liver where wbc engulfment was most apparent (bottom row). Body temperatures of hu-NSGS were significantly elevated relative to nonengrafted controls (Figure $1 \mathrm{~J}$ ). We also found a significant increase in sCD25 (IL-2R) in the serum of hu-NSGS mice (Figure $1 \mathrm{~K}$ ). Taken together, these data all strongly suggest a xenogeneic model of secondary HLH or MAS.

Anemic NSGS fail to respond to lymphocyte ablation. HLH and MAS are often treated with antilymphocyte therapies such as steroids, immunoglobulins, etoposide, and cyclosporine directed mainly toward controlling $\mathrm{CD}^{+} \mathrm{T}$ cells. We have previously shown that the anti-T cell antibody OKT3 is capable of specifically eliminating human T cells from xenografts (25), so we employed this method to attempt a cure in anemic hu-NSGS mice. Surprisingly, repeated dosing with OKT3, rituximab (antiCD20, targeting B cells), or both did not correct or slow the progressive anemia (Figure 2A). Dexamethasone treatment was similarly ineffective. Combined rituximab/OKT3 treatment led to a further reduction of wbc in the blood compared with control, which is consistent with the depletion of human $\mathrm{B}$ and $\mathrm{T}$ cells from the periphery (PBS $(n=13) 1.73 \mathrm{k} / \mu 1 \pm 0.82 \mathrm{vs}$. R/O $(n=14) 1.02 \mathrm{k} / \mu 1 \pm 0.34$; $P=0.0172$ by Mann-Whitney $U$ test). Platelet numbers were statistically unimproved in these mice (PBS $624 \pm 209 \mathrm{k} / \mu 1$ vs. R/O $707 \mathrm{k} / \mu 1 \pm 172$ ). To determine whether this failure was due to treating an out-of-control disease too late in the process, we treated preanemic mice with combined OKT3-rituximab therapy beginning just 1 day after engraftment to completely ablate lymphocyte production in hu-NSGS mice. Despite the total ablation of B and T lymphocytes from the mice, disease development was not altered in the treated mice relative to PBS control, demonstrating a lymphocyte-independent etiology (Figure 2B and data not shown). 
A

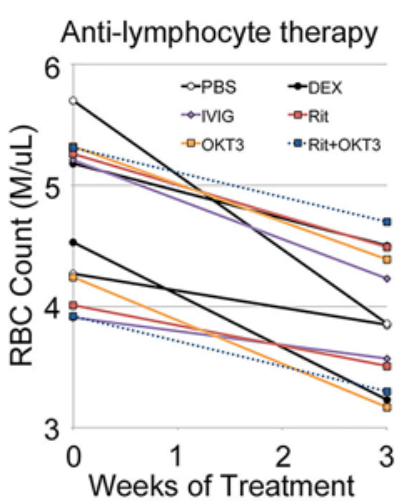

B

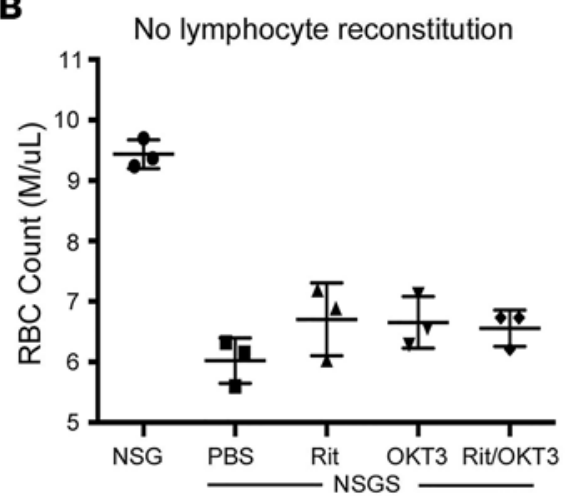

C

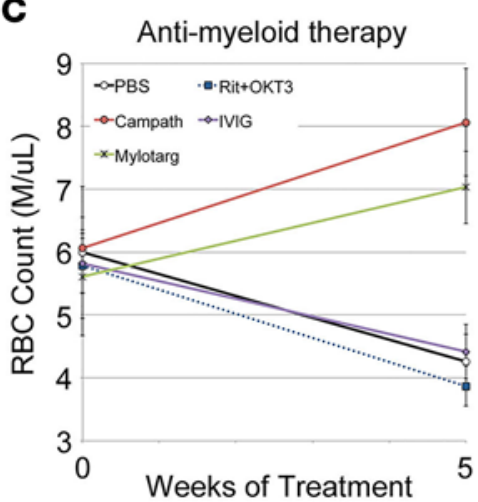

D

PBS

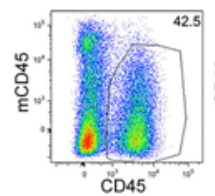

Specific antibody ablation

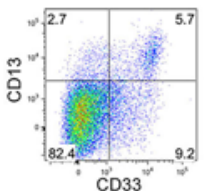

Campath

Rit +

OKT3
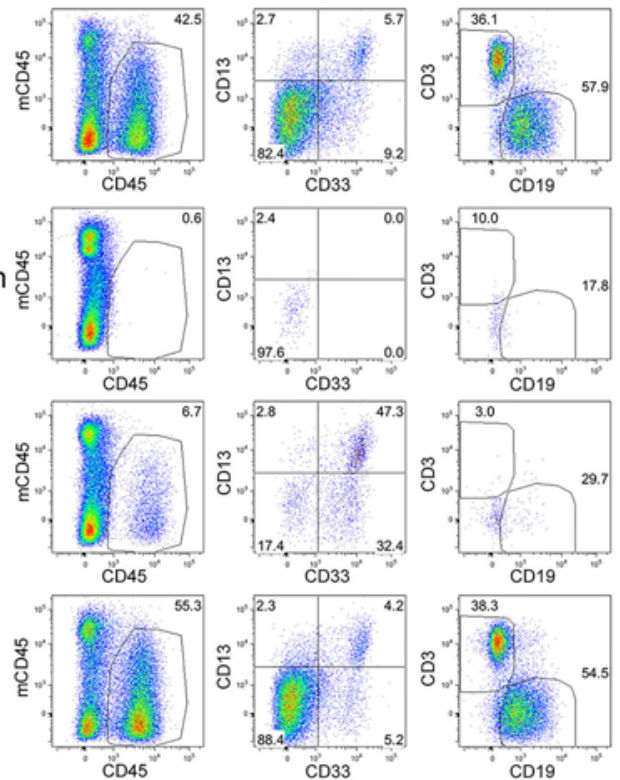

IVIG
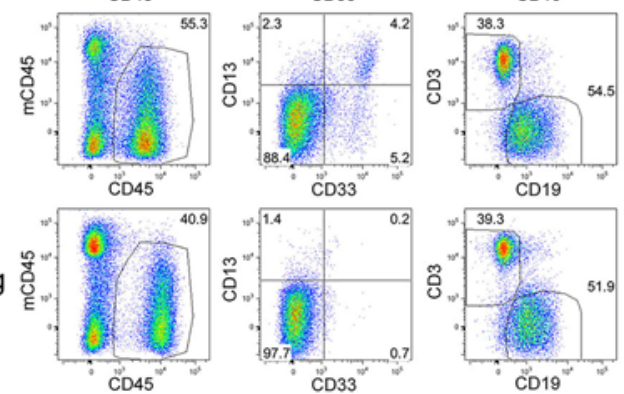

Mylotarg

CD19
E

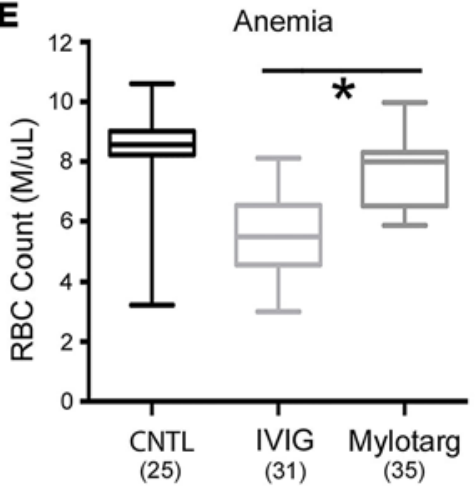

$\mathbf{F}$

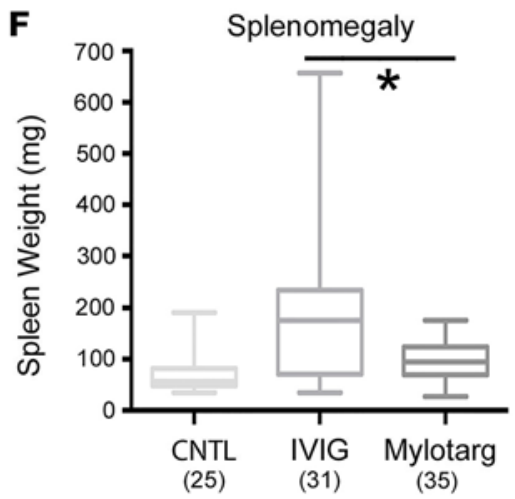

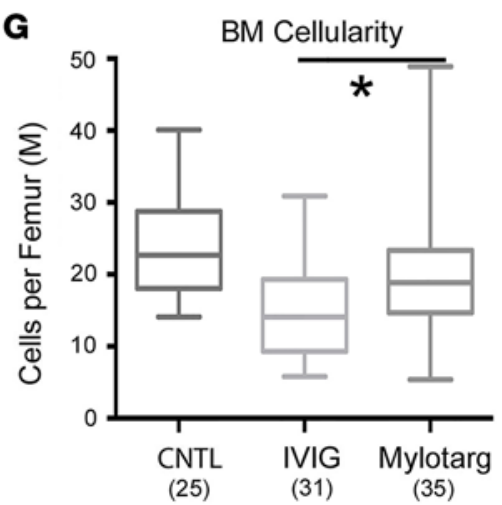

Figure 2. NSGS MAS is a lymphoid-independent, myeloid-driven disease. (A) Engrafted NSCS mice with MAS were treated with weekly doses of antibodies or steroids to ablate or inhibit human B and T cell activities (IVIG, i.v. Ig; Rit, rituximab; DEX, dexamethasone). The rbc counts before and after are shown for each mouse in a representative experiment. Each treatment was repeated in additional experiments at least 3 times. (B) Newly engrafted mice were given combinations of Rit and OKT3 to prevent B and T cell reconstitution in NSGS mice. The rbc counts were determined 12 weeks after engraftment. Three mice per group were used in this representative experiment. Mean \pm SD and individual mice are shown. (C) The experiment in $\mathbf{A}$ was repeated to include Campath and Mylotarg. Each treatment was repeated in at least 3 experiments. (D) Spleen preparations from antibody-treated mice were subjected to flow cytometry to demonstrate the degree and specificity of monoclonal antibody depletion in vivo. (E) PB rbc counts from engrafted mice or nonengrafted controls (CNTL). (F) Spleen weights and (C) BM cellularity were determined in the same mice as $\mathbf{E}$. ${ }^{*} P<0.05$ by Mann-Whitney $U$ test. Panels $\mathbf{E}, \mathbf{F}$, and $\mathbf{G}$ are box and whisker plots with the bounds of the box indicating the 1st and 3rd quartiles, the line within the box showing the mean, and the whiskers comprising the range of the data points.

Mice improve with direct targeting of human myeloid cells. The CD52 monoclonal antibody alemtuzumab (Campath) has been used with success as salvage therapy in cases of refractory and secondary HLH, whose disease manifestations mimic those of MAS $(26,27)$. In contrast to anemic mice that received dexamethasone, immunoglobulin, or anti-B/T antibodies, mice that received Campath showed a rapid, full recovery of rbc counts and disease manifestations (Figure 2C). However, when we examined BM and spleen for 
A

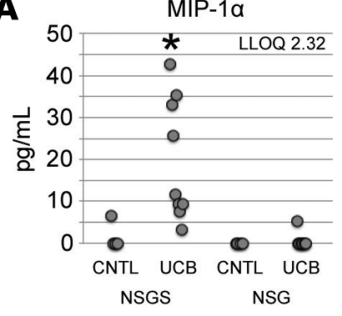

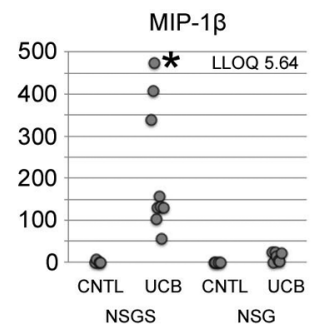
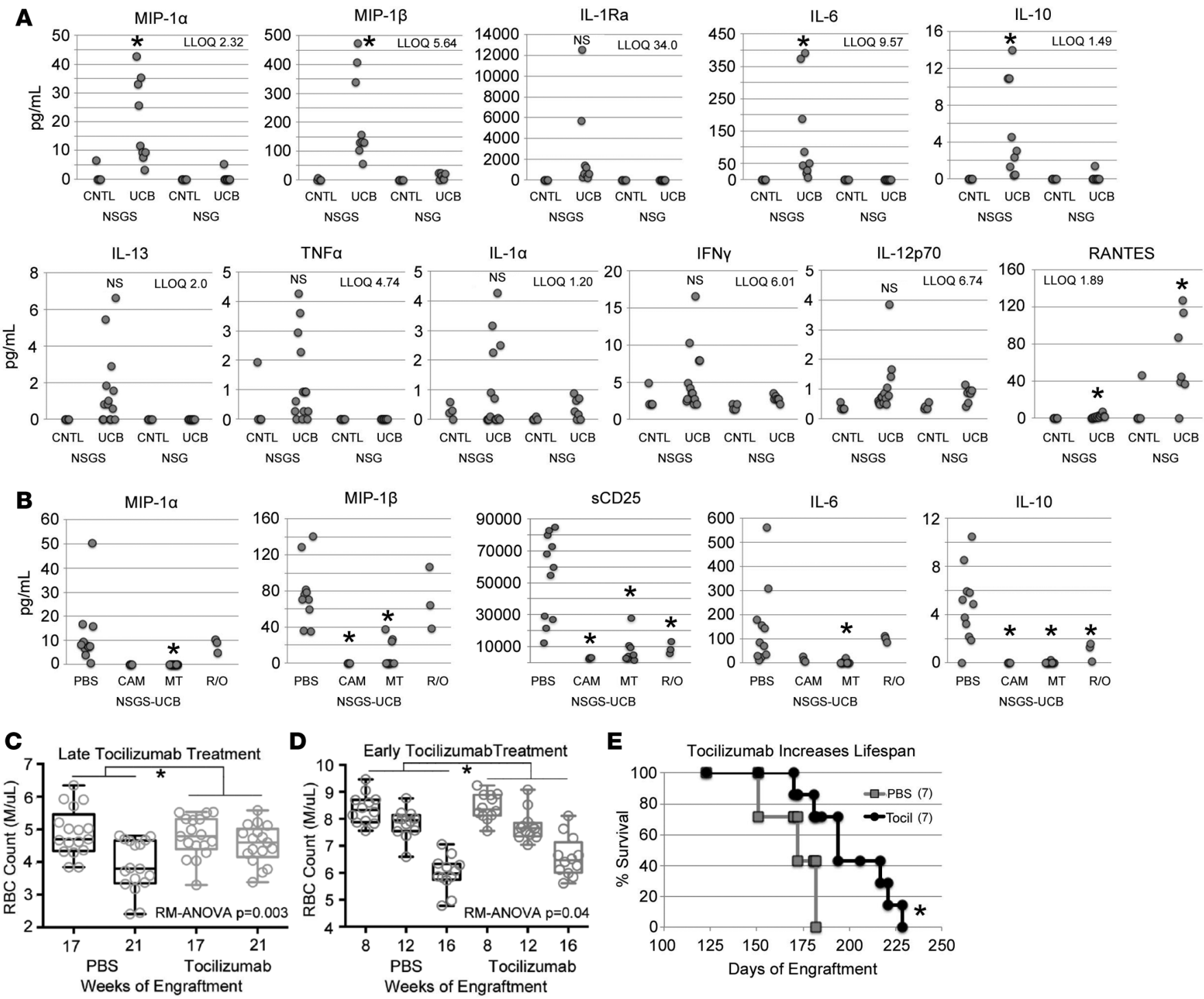

Figure 3. Identification and efficacy of targeting IL-6/IL-6R signaling. (A) Levels of several human inflammatory cytokines were measured in the plasma of control nonengrafted (CNTL) or long-term UCB-engrafted NSG and NSCS mice by multiplex ELISA. $n=4$ CNTL NSCS, $n=9-13$ UCB-NSCS, $n=4$ CNTL NSG, and $n=7$ UCB-NSG samples were used for analysis. LLOQ, lower limit of quantification. ${ }^{*} P<0.05$ by Mann-Whitney $U$ test. (B) Plasma levels of human cytokines in UCB-engrafted NSCS mice after the indicated treatments. CAM, Campath/alemtuzumab; MT, Mylotarg/gemtuzumab ozogamicin; R/O, rituximab + OKT3. $n=11 \mathrm{PBS}, n=3$ CAM, $n=9 \mathrm{MT}$, and $n=3 \mathrm{R} / 0$. One-way ANOVA followed by Tukey HSD test was used to determine significance. (C) Tocilizumab treatment was delayed until the mice had significant loss in PB rbc counts. Treatment started after the week 17 baseline measurement. $n=17$ mice per group. (D) UCB-engrafted NSCS were treated with PBS or tocilizumab (Tocil) before anemia could be detected. Serial blood counts were taken at the indicated times. Treatment started after the week 8 baseline measurement. $n=10$ mice per group. Panels $\mathbf{C}$ and $\mathbf{D}$ are box and whisker plots with the bounds of the box indicating the 1st and 3rd quartiles, the line within the box showing the mean, and the whiskers comprising the range of the data points. Repeated measures 2-way ANOVA was performed on the data in $\mathbf{C}$ and $\mathbf{D}$ to determine significance. (E) Treated mice were followed long-term for survival. The log rank test was used to determine differences in survival.

engraftment, we found a complete lack of human cells (Figure 2D). Further examination confirmed that all hematopoietic cells of human xenografts stain positive using a CD52 flow antibody (data not shown). Therefore, Campath eliminates the entire graft and not just the human lymphocytes. While this result does not help to identify the specific effector cell(s) driving the disease, it does show that the human graft is responsible and that the disease is reversible.

We also tested a CD33 targeted myeloablating chemotherapy, gemtuzumab ozogamicin (Mylotarg, MT), which has previously been used to treat acute myeloid leukemia (28). The rbc counts rebounded after MT treatment, and analysis showed specific depletion of the myeloid but not the lymphoid component of the graft (Figure 2, C-E). Additionally, MT-treated mice showed marked improved in appearance, 
splenomegaly was nearly corrected, and BM cellularity improved significantly (Figure 2, F and G, and data not shown). These data demonstrate a myeloid-driven pathology and strongly suggest MAS rather than lymphocyte-driven secondary HLH.

Tocilizumab delays disease progression. Many studies have investigated the cytokine profiles of patients with secondary HLH and MAS. Using multiplex ELISA assays, we analyzed the serum from mice with active disease in order to determine which human cytokines were expressed. While many cytokines were very low (IFN $\gamma$, IL-12p70, IL-13, RANTES, TNF $\alpha$, IL-1A) or negative (IL-23, M-CSF, MMP-7, MMP-1, IL-1B, IL-4) in the majority of samples, we identified several cytokines (MIP-1A, MIP-1B, IL-1Ra, IL-6, IL-10) specifically increased in hu-NSGS with active disease relative to healthy hu-NSG (Figure 3A). Control sera from nonhumanized mice demonstrated the specificity of the antibodies for human cytokines. To correlate cytokine expression with disease status, we tested sera from hu-NSGS that were cured of disease due to total graft eradication (Campath) or specific ablation of human myeloid cells (MT), as well as diseased hu-NSGS ablated for lymphocytes (rituximab/OKT3). IL-1Ra levels disappeared with all treatments, including rituximab/OKT3, which did not affect disease phenotype and therefore seems less relevant in our model (Figure 3B). In contrast, MIP-1A, MIP-1B, and IL-6 were decreased upon MT or Campath therapy but not with rituximab/OKT3 $(\mathrm{R} / \mathrm{O})$ treatment, indicating that a human myeloid cell is likely the cell responsible for production of these cytokines. Interestingly, IL-10 was significantly decreased in sera from all 3 treatment groups. It is possible that one or more of these signals is important for disease initiation and/or progression and may represent a target for therapeutic intervention.

IL-6 is a target that is being pursued in the clinic. A clinical trial is underway to examine the efficacy of tocilizumab, a monoclonal antibody against the IL-6 receptor, as an adjunct therapy for HLH (www.clinicaltrials.gov, identifier NCT02007239). This antibody has also shown some success in 2 recent case reports of secondary HLH induced by disparate triggers $(29,30)$. To determine efficacy of tocilizumab in diseased hu-NSGS mice, we treated anemic mice and found a slowing of the progressive drop in rbc counts, which were significant after several weeks of therapy (Figure 3C). We did not find any significant changes in wbc or platelet numbers in the PB of tocilizumab-treated mice relative to PBS injected controls. We observed similar effects in mice that were treated early after engraftment, before anemia was detectable (Figure 3D). Additionally, the treated mice showed a marked improvement in appearance, and tocilizumab treatment resulted in a significant extension in lifespan, indicating a modest benefit of this approach in this model (Figure 3E).

\section{Discussion}

Here, we present a xenograft model of MAS. Establishment of a human immune cell graft in NSGS mice resulted in progressive disease with many of the hallmarks of secondary HLH and MAS. Disease manifestation was reversible upon graft eradication with Campath or by specific ablation of human myeloid but not of lymphoid cells. A number of inflammatory cytokines tracked with disease status, and targeted therapy using tocilizumab was effective in slowing disease progression and significantly extending the life of affected mice. This model will be useful to explore the mechanisms of disease initiation and progression and has utility for preclinical testing of novel therapies. For example, it will be very interesting to follow up on the success demonstrated here with gemtuzumab ozogamicin and identify additional targetable molecules on the surface of the myeloid effectors. An ideal target would be more selective than CD33 or specific to the aberrant hyperactive state found in MAS. Such an approach could be invaluable in combating cases that are refractory to currently available therapies.

While MAS was readily apparent in the NSGS/NRGS strains, it was notably absent in NSS mice, which also contain the transgenic cytokines but retain the common $\gamma$ chain. On the cellular level, a major consequence is that NSS mice retain functional endogenous NK cells, which may exert enough immune activity to suppress the expansion or function of the necessary human myeloid cells. NK activity has long been believed to be a barrier to human engraftment in NOD/SCID mouse strains. This can be overcome by genetic inactivation or antibody depletion of murine NK cells $(31,32)$. Using a genetic BM transplant model, a recent report has shown that functional NK cells were sufficient to protect $\mathrm{Prf1}^{-/-}$BM-transplanted mice from many of the features of LCMV induced HLH (33). Consistent with our findings, this model also observed HLH development in $\mathrm{Rag}^{-/-} \mathrm{IL}^{2} \mathrm{rg}^{-/-}$recipient mice, but not in Rag2 $2^{-/}$IL $2 \mathrm{rg}^{+/+}$recipient mice. Additional relevant findings relating to our model were that NK protection was IFN $\gamma$ independent and was associated with reduced inflammatory macrophage tissue infiltration. 
One other xenograft model has been described that utilizes UCB engraftment into immune deficient mice (10). In this model, utilizing the NOD/SCID-IL2R $\gamma^{\text {(truncated) }}$ mouse (NOG, very similar to NSG), a majority of the mice developed fatal HLH by 10 weeks after infection with EBV and exhibited significant expansion of $\mathrm{T}$ cells, increased IFN $\gamma$, and infiltration of $\mathrm{T}$ and phagocytic myeloid cells into numerous tissues. This model built on previous reports of recapitulation of HLH phenotypes in NOD/SCID mice upon transplant of herpesvirus saimiri -transformed (HVS-transformed) T cell lines generated from HLH patients (34) or in NOG mice after transfer of peripheral blood mononuclear cells (PBMNCs) from EBV-infected HLH patients (35). Our model is markedly different than these in that the macrophage cells are activated independently of lymphocytes and the latter are dispensable for the phenotype (Figure 2). Instead, mice showed a remarkable recovery upon treatment with a clinically relevant myeloid-depleting antibody.

Consistent with the lack of a requirement for $\mathrm{T}$ cells in our model, we observed a relatively low level of IFN $\gamma$, a central component in other models of HLH. Recent studies have shown that the role of lymphocyte-derived IFN $\gamma$ may not always be required in the initiation of inflammation, particularly in models of secondary HLH and MAS. There are sporadic cases without increased levels of IFN $\gamma$ (36). Direct activation of innate immune cells via TLR9 activation was dependent on IFN $\gamma$ but did not require lymphocytes, implicating an alternative source of IFN $\gamma$ in this model (12). Subsequently, it was shown that TLR9 activation, along with IL-10 inhibition, completely bypasses the requirement for IFN $\gamma$ for fulminant MAS, demonstrating the potential for noncanonical mechanisms of MAS initiation (37). Further evidence comes from a recent study of CMV-induced secondary HLH in BALB/c mice (11). In this approach, not only did depletion of $\mathrm{CD}^{+} \mathrm{T}$ cells not affect disease, but IFN $\gamma$-deficient mice actually developed a more robust form of HLH. Additionally, a mutation in NLRC4 was shown to result in activation of macrophage inflammasomes and secretion of high levels of IL-1 $\beta$ and IL-18, which led to MAS without increased IFN $\gamma$ or lymphocyte cytotoxic defects (38). Macrophage cells from patients with chronic granulomatous disease, a group susceptible to MAS, have been shown to have a similar activation of inflammasomes and IL-1 $\alpha$ and IL- $1 \beta$ release resulting from mutations that cause NADPH oxidase inactivation (39). These findings in patients highlight the possibility that myeloid intrinsic defects can drive the disease.

There is substantial evidence that dysregulated GM-CSF signaling contributes directly to pathogenic inflammatory conditions. Transgenic GM-CSF mice displayed increases in inflammatory cytokines and macrophage accumulation and activation resulting in significant, fatal tissue damage (40), while more focused transgenic expression led to autoimmune gastritis (41). Injection of GM-CSF into mice worsened collagen-induced arthritis (42), while - on the other hand - GM-CSF KO mice are resistant to induction of arthritis (43). The GM-CSF pathway has also been shown to play a role in rheumatoid arthritis (RA) and has recently been studied as a potential therapeutic target. The GM-CSFR $\alpha$ monoclonal antibody mavrilimumab has shown promise in clinical trials to treat RA (44). This is a substantial shift in therapy in that traditional treatment has focused on control of the lymphocytes and their activities (TNF $\alpha$ signaling), while mavrilimumab aims to target the effector cell directly. Targeting GM-CSF for blockade is a promising strategy for a number of autoimmune and inflammatory disorders (45). These disorders are likely to have downstream pathways in common with HLH and MAS. Our model of secondary HLH/MAS will allow a dissection of these downstream signals and permit the testing of therapeutic targeting strategies.

Additional information comes from mouse models with overexpression of IL-3 and SCF. When murine IL-3 expression was unrestricted from a CMV promoter, an ALS-like state was observed due to autoimmune activity against spinal cord motor neurons, which led to hind-limb paralysis and death at around 10 months of age (46). Similarly, cerebral restricted expression of murine IL-3 using a glial fibrillary acidic protein (GFAP) promoter also resulted in neurological defects; however, the observed phenotypes were more MS-like and were correlated with inflammation and recruitment of macrophages, leading to demyelination (47). These earlier studies highlight the ability of IL-3 to provoke inappropriate hematopoietic cell responses. A study of a human SCF transgenic mouse found that hSCF decreased c-kit (the SCF receptor) and interfered with murine SCF/c-kit signaling without inducing signaling through c-kit (48). Similar to our model, a membrane-bound human SCF causes anemia and increased myeloid engraftment when expressed in NSG mice (49), a phenotype that is reminiscent of that observed in mice with loss of function SCF alleles (50). It is unclear how the high levels of soluble human SCF in our model might affect erythropoiesis, however, given that membrane associated and soluble forms of SCF may have differential activities (51). It is quite possible that the MAS that develops in our model may not be easily attributable to a single cytokine but may rather be the result of a mixture of all 3 , with distinct and overlapping effects on both human myeloid differentiation and function, as well as murine erythropoiesis. 
JAK inhibition has recently been identified as a potential therapy for primary and secondary HLH (15). Interestingly, the study of NLRC4-MAS identified increased levels of both IL-3 and SCF in the serum of those patients (38). While it is unclear which of the transgenic human cytokines is key for MAS development in our model, both IL-3 and GM-CSF signal through the JAK/STAT pathway, suggesting that our model may be ideal to assess the utility of such a therapy.

Screening of long-term hu-NSGS mice for evidence of inflammatory cytokines associated with HLH identified several relevant proteins. MIP- $1 \alpha$ and $-\beta$ (CCL3/CCL4) chemokines were specifically elevated, and their levels correlated with diseases status. These chemokines play key roles in attracting immune cells to areas of inflammation and infection, although their specific role in HLH/MAS is currently unknown. It would be of interest to determine whether neutralization of these proteins would partially interfere with the unfettered cycle of inflammation and ameliorate any of the disease phenotypes. Elevated serum IL-10 is a highly specific and accurate biomarker for the diagnosis of $\mathrm{HLH}$, particularly when combined with elevated IFN $\gamma$ and moderate IL-6 (52). In the current model, elevated IL-10 was present and the levels decreased upon disease eradication. Interestingly, IL-10 levels decreased with either myeloid or lymphoid cell ablation, indicating that both populations are central to IL-10 production. This blunted IL-10 production after lymphoid ablation is reminiscent of that seen in the TLR9 MAS model when B/T/NK-deficient Rag2 $2^{-/}$mice were used (12). While monocytes likely produce the bulk of the IL-10, some lymphocytes, such as peritoneal B cells, have been shown to produce IL-10 upon TLR9 stimulation (53). However, given that IL-10 is a key negative regulator of macrophage activity, it is unclear what role it may play in disease pathogenesis, and it is unlikely to be a therapeutic target for the treatment of HLH (54).

Inhibition of IL-6 signaling with tocilizumab, an anti-IL-6R monoclonal antibody, has shown dramatic results in the successful control of RA and in the treatment of other disorders associated with increased levels of IL-6 $(55,56)$. IL-6R inhibition with tocilizumab is an attractive option for combating cytokine release syndrome, a state that closely resembles MAS/HLH, which can develop following treatment with chimeric antigen receptor-modified T cell or bispecific T cell engager therapies for leukemia. (29, 57). Early results demonstrate efficacy while minimizing the risk of $\mathrm{T}$ cell inactivation that comes with other options (58). Tocilizumab has also shown some promise in secondary HLH and is being tested in a clinical trial as an adjunct therapy for $\operatorname{HLH}(29,30)$. The results we obtained in the current study would indicate there may be a role for tocilizumab in controlling secondary HLH/MAS. Interestingly, a study of tocilizumab for patients with SoJIA observed the appearance of MAS in approximately $4 \%$ of the cohort, similar to the reported frequency of MAS found in SoJIA patients not receiving tocilizumab (59, 60). Similarly, SoJIA patients treated with other biologic therapies including canakinumab, etanercept, and anakinra showed a low frequency of MAS development while on therapy, indicating that single cytokine inhibition may not be sufficient to prevent the development of MAS. Whether these therapies are able to affect the severity of disease, as shown for tocilizumab in the present study, remains to be examined. In addition, the question of whether combinations of agents are more effective than single-agent therapy should be experimentally tested. The model presented in this study affords a platform in which to pursue such studies.

\section{Methods}

Xenografts. NS, NSG, and NRG mice were obtained from Jackson Laboratories. NSS (24), NSGS (21), and NRGS (25) are derivative strains that express human SCF, GM-CSF, and IL-3. Mice were bred, housed, and used in a pathogen-free facility at CCHMC according to standard procedures. Mice were conditioned with a single $30 \mathrm{mg} / \mathrm{kg}$ i.p. dose of busulfan 24 hours before tail vein injection of OKT3-treated unfractionated UCB cells, as described (25). Serial CBC analysis was performed on PB collected from tail veins using a HemaVet 9500 (Drew Scientific). Reticulocytes were determined with the Retic-Count reagent according to the manufacturer's recommendations (BD Biosciences). Whole BM cellularity was calculated from HemaVet-generated wbc counts after crushing bones in a set volume of buffer with a mortar and pestle. Bone samples were stored in $10 \%$ formalin prior to paraffin embedding and staining with H\&E. Rectal temperatures were taken from active mice with a model BAT-12 physitemp instrument (Physitemp Instrument Inc.).

Cytospins and microscopy. Cells $\left(2 \times 10^{4}\right.$ to $\left.6 \times 10^{4}\right)$ in $200 \mu \mathrm{PBS} / 2 \%$ FBS from single-cell preparations of BM, spleen, and liver were added to Shandon Single Cytofunnels (Thermo Scientific) clipped to glass slides and centrifuged using a Cytospin 4 centrifuge (Thermo Scientific) set at $500 \mathrm{rpm}$ ( $28 \mathrm{~g}$ ) for $5 \mathrm{~min}$ utes at medium acceleration. After drying, cells were stained with Protocol Wright Giemsa Stain (Fisher 
Scientific) according to the manufacturer's suggested procedure. Images were acquired with NIS Elements software from a Nikon Eclipse 80i microscope equipped with a Nikon DS-Fi1 camera.

Transfusions. Donor mice were bled from tail veins into $1.5-\mathrm{ml}$ tubes containing $24 \mathrm{U}$ of heparin and EDTA at a final concentration of $2 \mathrm{mM}$. Pooled donor blood $(350 \mu \mathrm{l})$ was immediately injected into the tail veins of recipient mice. For CFSE experiments, blood was washed with PBS $/ 2 \%$ FBS and then incubated for 1 hour at $37^{\circ} \mathrm{C}$ in $10 \mu \mathrm{M}$ CFSE (Invitrogen) before being rewashed and infused into recipient mice.

Treatment of anemic mice. Mice were treated with weekly $10 \mathrm{mg} / \mathrm{kg}$ i.p. doses of i.v. immunoglobulin (IV/IG, Gammagard, Baxter Healthcare) or monoclonal antibodies against human CD20 (rituximab, Rituxan, Genentech), CD52 (alemtuzumab, Campath, Genzyme Corporation), and/or CD3 (OKT3, catalog BE0001-2, BioXcell). Tocilizumab (anti-IL-6R, Actemra, Genentech) was given twice per week. IV/IG, rituximab, alemtuzumab, and tocilizumab were obtained from the residual unused portion of single-use vials used clinically at CCHMC. Gemtuzumab ozogamicin (GO, MT, Wyeth/Pfizer) was a gift from May Kung Sutherland (Seattle Genetics, Seattle, Washington, USA) and was used at $0.1 \mathrm{mg} / \mathrm{kg}$. All antibodies were diluted in PBS containing 2\% FBS. Dexamethasone (APP Pharmaceuticals) was given at $1 \mathrm{mg} / \mathrm{kg}$, i.p.

Flow cytometry. Spleen preparations were stained with antibodies in PBS $/ 3 \%$ FBS at $4^{\circ} \mathrm{C}$ for 2 hours. Antibodies were against mouse CD45 (APC-Cy7, BD Biosciences, catalog 557659) and against human CD45 (FITC, BD Biosciences, catalog 555482), CD13 (PE, BD Biosciences, catalog 555394), CD33 (APC, BD Biosciences, catalog 551378), CD3 (PE-Cy7, BD Biosciences, catalog 557851), and CD19 (VioBlue, Miltenyi Biotec, catalog 130-098-598). Anti-Fc $\gamma$ R antibodies were also added against mouse (Miltenyi Biotec, catalog 120-003-855) and human (Miltenyi Biotec, catalog 120-000-442). After washing, samples were assessed with BD FACSCanto machines and data was analyzed with FloJo software (Tree Star Inc.).

Multiplex ELISA. Peripheral blood samples were obtained from mice at sacrifice by cardiac puncture and were collected in EDTA tubes. Plasma samples were prepared by centrifugation at 15,000 $\mathrm{g}$ for $10 \mathrm{~min}$ utes. Samples were stored at $-80^{\circ} \mathrm{C}$ until analyzed. All ELISA results were obtained from custom ProcartaPlex panels created by combining multiple simplex bead sets. Assays were run using the Procarta Human Basic Kit (eBioscience, catalog EPX010-10420-901). A separate single simplex bead kit was used alone for the sCD25 data presented in Figures 1 and 3.

Statistics. Statistics were performed with GraphPad Prism 6 software. Unless specified below, significance was determined by Mann-Whitney $U$ analysis. $P<0.05$ was used as a cut off for significance. For comparison of multiple treatment groups in Figure 3B, one-way ANOVA was performed, followed by Tukey tests. For differences in survival, the log rank test was used. In Figures 3, C and D, the 2-way repeated measures ANOVA was performed.

Study approval. Animal handling is according to the NIH and the Association for Assessment and Accreditation of Laboratory Animal Care (AAALAC) and experiments were performed under a protocol approved by the IRB of CCHMC. UCB samples were obtained from the Translational Trials Development Support Laboratories of CCHMC after informed consent and are also approved by the CCHMC IRB.

\section{Author Contributions}

MW designed the study and experiments, performed experiments, analyzed and interpreted data, and wrote the manuscript. C. Stockman, MD, and NR performed experiments and analyzed data. C. Sexton performed experiments. BM performed experiments, analyzed data, and assisted with the design of experiments. ARK interpreted data and edited the manuscript. JCM designed the study and experiments, interpreted data, and wrote the manuscript.

\section{Acknowledgments}

We thank the Flow Cytometry Core at CCHMC for their help. We thank Alexei Grom, Michael Jordan, and Kimberly Risma of CCHMC for helpful discussions and suggestions, Kasiani Myers for coordinating transfer of surplus clinical therapeutics for research, and Alyssa Sproles for assistance with multiplex ELISA assays. This work was supported by an Institutional Clinical and Translational Science Award, NIH/NCRR grant 1UL1RR026314-01, Translational Trials Development and Support Laboratory award (USPHS grant MO1 RR 08084), and a Center of Excellence in Molecular Hematology P30 award (DK090971). BM is a St. Baldrick's Foundation Scholar. JCM is a Leukemia and Lymphoma Society Scholar. 
Address correspondence to: Mark Wunderlich or James C. Mulloy, Cancer and Blood Disease Institute, Division of Experimental Hematology and Cancer Biology, MLC 7013, Cincinnati Children's Hospital Medical Center, 3333 Burnet Avenue, Cincinnati, Ohio 45229, USA. Phone: 513.803.1983; E-mail: mark.wunderlich@ cchmc.org (M. Wunderlich). Phone: 513.636.1844; E-mail: james.mulloy@cchmc.org (J.C. Mulloy).

1. Grom AA, Mellins ED. Macrophage activation syndrome: advances towards understanding pathogenesis. Curr Opin Rheumatol. 2010;22(5):561-566.

2. Sawhney S, Woo P, Murray KJ. Macrophage activation syndrome: a potentially fatal complication of rheumatic disorders. Arch Dis Child. 2001;85(5):421-426

3. Bleesing J, et al. The diagnostic significance of soluble CD163 and soluble interleukin-2 receptor alpha-chain in macrophage activation syndrome and untreated new-onset systemic juvenile idiopathic arthritis. Arthritis Rheum. 2007;56(3):965-971.

4. Behrens EM, Beukelman T, Paessler M, Cron RQ. Occult macrophage activation syndrome in patients with systemic juvenile idiopathic arthritis. J Rheumatol. 2007;34(5):1133-1138.

5. Atteritano M, et al. Haemophagocytic syndrome in rheumatic patients. A systematic review. Eur Rev Med Pharmacol Sci. 2012;16(10):1414-1424.

6. Schulert GS, Grom AA. Macrophage activation syndrome and cytokine-directed therapies. Best Pract Res Clin Rheumatol. 2014;28(2):277-292.

7. Canna SW, Behrens EM. Not all hemophagocytes are created equally: appreciating the heterogeneity of the hemophagocytic syndromes. Curr Opin Rheumatol. 2012;24(1):113-118.

8. Brisse E, Wouters CH, Matthys P. Hemophagocytic lymphohistiocytosis (HLH): A heterogeneous spectrum of cytokine-driven immune disorders. Cytokine Growth Factor Rev. 2015;26(3):263-280.

9. Jordan MB, Hildeman D, Kappler J, Marrack P. An animal model of hemophagocytic lymphohistiocytosis (HLH): CD8+ T cells and interferon gamma are essential for the disorder. Blood. 2004;104(3):735-743.

10. Sato K, et al. A novel animal model of Epstein-Barr virus-associated hemophagocytic lymphohistiocytosis in humanized mice. Blood. 2011;117(21):5663-5673.

11. Brisse E, et al. Mouse Cytomegalovirus Infection in BALB/c Mice Resembles Virus-Associated Secondary Hemophagocytic Lymphohistiocytosis and Shows a Pathogenesis Distinct from Primary Hemophagocytic Lymphohistiocytosis. J Immunol. 2016;196(7):3124-3134.

12. Behrens EM, et al. Repeated TLR9 stimulation results in macrophage activation syndrome-like disease in mice. J Clin Invest. 2011;121(6):2264-2277.

13. Pachlopnik Schmid J, et al. Neutralization of IFNgamma defeats haemophagocytosis in LCMV-infected perforin- and Rab27a-deficient mice. EMBO Mol Med. 2009;1(2):112-124.

14. Chiossone L, et al. Protection from inflammatory organ damage in a murine model of hemophagocytic lymphohistiocytosis using treatment with IL-18 binding protein. Front Immunol. 2012;3:239.

15. Das R, et al. Janus kinase inhibition lessens inflammation and ameliorates disease in murine models of hemophagocytic lymphohistiocytosis. Blood. 2016;127(13):1666-1675.

16. Shultz LD, et al. Human lymphoid and myeloid cell development in NOD/LtSz-scid IL2R gamma null mice engrafted with mobilized human hemopoietic stem cells. J Immunol. 2005;174(10):6477-6489.

17. Ishikawa F, et al. Development of functional human blood and immune systems in NOD/SCID/IL2 receptor \{gamma\} chain(null) mice. Blood. 2005;106(5):1565-1573.

18. Ito M, et al. NOD/SCID/gamma(c)(null) mouse: an excellent recipient mouse model for engraftment of human cells. Blood 2002;100(9):3175-3182.

19. Yahata T, et al. Functional human T lymphocyte development from cord blood CD34+ cells in nonobese diabetic/Shi-scid, IL-2 receptor gamma null mice. J Immunol. 2002;169(1):204-209.

20. Miller PH, et al. Enhanced normal short-term human myelopoiesis in mice engineered to express human-specific myeloid growth factors. Blood. 2013;121(5):e1-e4.

21. Wunderlich M, et al. AML xenograft efficiency is significantly improved in NOD/SCID-IL2RG mice constitutively expressing human SCF, GM-CSF and IL-3. Leukemia. 2010;24(10):1785-1788.

22. Coughlan AM, et al. Myeloid Engraftment in Humanized Mice: Impact of Granulocyte-Colony Stimulating Factor Treatment and Transgenic Mouse Strain. Stem Cells Dev. 2016;25(7):530-541.

23. Billerbeck E, Barry WT, Mu K, Dorner M, Rice CM, Ploss A. Development of human CD4+FoxP3+ regulatory T cells in human stem cell factor-, granulocyte-macrophage colony-stimulating factor-, and interleukin-3-expressing NOD-SCID IL2R $\gamma$ (null) humanized mice. Blood. 2011;117(11):3076-3086.

24. Nicolini FE, Cashman JD, Hogge DE, Humphries RK, Eaves CJ. NOD/SCID mice engineered to express human IL-3, GM-CSF and Steel factor constitutively mobilize engrafted human progenitors and compromise human stem cell regeneration. Leukemia. 2004;18(2):341-347.

25. Wunderlich M, Brooks RA, Panchal R, Rhyasen GW, Danet-Desnoyers G, Mulloy JC. OKT3 prevents xenogeneic GVHD and allows reliable xenograft initiation from unfractionated human hematopoietic tissues. Blood. 2014;123(24):e134-e144.

26. Marsh RA, et al. Salvage therapy of refractory hemophagocytic lymphohistiocytosis with alemtuzumab. Pediatr Blood Cancer. 2013;60(1):101-109.

27. Keith MP, Pitchford C, Bernstein WB. Treatment of hemophagocytic lymphohistiocytosis with alemtuzumab in systemic lupus erythematosus. J Clin Rheumatol. 2012;18(3):134-137.

28. Sievers EL, et al. Selective ablation of acute myeloid leukemia using antibody-targeted chemotherapy: a phase I study of an anti-CD33 calicheamicin immunoconjugate. Blood. 1999;93(11):3678-3684.

29. Teachey DT, et al. Cytokine release syndrome after blinatumomab treatment related to abnormal macrophage activation and 
ameliorated with cytokine-directed therapy. Blood. 2013;121(26):5154-5157.

30. Rios-Fernandez R, Callejas-Rubio JL, Garcia-Rodriguez S, Sancho J, Zubiaur M, Ortego-Centeno N. Tocilizumab as an Adjuvant Therapy for Hemophagocytic Lymphohistiocytosis Associated With Visceral Leishmaniasis [published online ahead of print March 12, 2015]. Am J Ther. doi: 10.1097/MJT.0000000000000035.

31. Kollet $\mathrm{O}$, et al. beta2 microglobulin-deficient (B2m(null)) NOD/SCID mice are excellent recipients for studying human stem cell function. Blood. 2000;95(10):3102-3105.

32. McKenzie JL, Gan OI, Doedens M, Dick JE. Human short-term repopulating stem cells are efficiently detected following intrafemoral transplantation into NOD/SCID recipients depleted of CD122+ cells. Blood. 2005;106(4):1259-1261.

33. Sepulveda FE, et al. A novel immunoregulatory role for NK-cell cytotoxicity in protection from HLH-like immunopathology in mice. Blood. 2015;125(9):1427-1434.

34. Yoshida N, et al. Engraftment and dissemination of $\mathrm{T}$ lymphocytes from primary haemophagocytic lymphohistiocytosis in scid mice. Br J Haematol. 2003;121(2):349-358.

35. Imadome K, et al. Novel mouse xenograft models reveal a critical role of CD4+ T cells in the proliferation of EBV-infected T and NK cells. PLoS Pathog. 2011;7(10):e1002326.

36. Yasutomi M, et al. Cytokine profiles in Mycoplasma pneumoniae infection-associated hemophagocytic lymphohistiocytosi. J Microbiol Immunol Infect. 2014; pii:S1684-1182(14)00246-1.

37. Canna SW, Wrobel J, Chu N, Kreiger PA, Paessler M, Behrens EM. Interferon- $\gamma$ mediates anemia but is dispensable for fulminant toll-like receptor 9-induced macrophage activation syndrome and hemophagocytosis in mice. Arthritis Rheum 2013;65(7):1764-1775.

38. Canna SW, et al. An activating NLRC4 inflammasome mutation causes autoinflammation with recurrent macrophage activation syndrome. Nat Genet. 2014;46(10):1140-1146.

39. Meissner F, Seger RA, Moshous D, Fischer A, Reichenbach J, Zychlinsky A. Inflammasome activation in NADPH oxidase defective mononuclear phagocytes from patients with chronic granulomatous disease. Blood. 2010;116(9):1570-1573.

40. Lang RA, et al. Transgenic mice expressing a hemopoietic growth factor gene (GM-CSF) develop accumulations of macrophages, blindness, and a fatal syndrome of tissue damage. Cell. 1987;51(4):675-686.

41. Biondo M, Nasa Z, Marshall A, Toh BH, Alderuccio F. Local transgenic expression of granulocyte macrophage-colony stimulating factor initiates autoimmunity. J Immunol. 2001;166(3):2090-2099.

42. Campbell IK, Bendele A, Smith DA, Hamilton JA. Granulocyte-macrophage colony stimulating factor exacerbates collagen induced arthritis in mice. Ann Rheum Dis. 1997;56(6):364-368.

43. Campbell IK, Rich MJ, Bischof RJ, Dunn AR, Grail D, Hamilton JA. Protection from collagen-induced arthritis in granulocyte-macrophage colony-stimulating factor-deficient mice. J Immunol. 1998;161(7):3639-3644.

44. Di Franco M, Gerardi MC, Lucchino B, Conti F. Mavrilimumab: an evidence based review of its potential in the treatment of rheumatoid arthritis. Core Evid. 2014;9:41-48.

45. Wicks IP, Roberts AW. Targeting GM-CSF in inflammatory diseases. Nat Rev Rheumatol. 2016;12(1):37-48

46. Chavany C, Vicario-Abejón C, Miller G, Jendoubi M. Transgenic mice for interleukin 3 develop motor neuron degeneration associated with autoimmune reaction against spinal cord motor neurons. Proc Natl Acad Sci USA. 1998;95(19):11354-11359.

47. Chiang CS, Powell HC, Gold LH, Samimi A, Campbell IL. Macrophage/microglial-mediated primary demyelination and motor disease induced by the central nervous system production of interleukin-3 in transgenic mice. J Clin Invest. 1996;97(6):1512-1524.

48. Majumdar MK, et al. Xenogeneic expression of human stem cell factor in transgenic mice mimics codominant c-kit mutations. Blood. 1996;87(8):3203-3211.

49. Takagi S, et al. Membrane-bound human SCF/KL promotes in vivo human hematopoietic engraftment and myeloid differentiation. Blood. 2012;119(12):2768-2777.

50. Kapur R, Cooper R, Xiao X, Weiss MJ, Donovan P, Williams DA. The presence of novel amino acids in the cytoplasmic domain of stem cell factor results in hematopoietic defects in Steel(17H) mice. Blood. 1999;94(6):1915-1925.

51. Kapur R, Majumdar M, Xiao X, McAndrews-Hill M, Schindler K, Williams DA. Signaling through the interaction of mem brane-restricted stem cell factor and c-kit receptor tyrosine kinase: genetic evidence for a differential role in erythropoiesis. Blood. 1998;91(3):879-889.

52. Xu XJ, et al. Diagnostic accuracy of a specific cytokine pattern in hemophagocytic lymphohistiocytosis in children. $J$ Pediatr. 2012;160(6):984-90.e1.

53. Sindhava V, Woodman ME, Stevenson B, Bondada S. Interleukin-10 mediated autoregulation of murine B-1 B-cells and its role in Borrelia hermsii infection. PLoS ONE. 2010;5(7):e11445.

54. Couper KN, Blount DG, Riley EM. IL-10: the master regulator of immunity to infection. J Immunol. 2008;180(9):5771-5777. 55. Shetty A, et al. Tocilizumab in the treatment of rheumatoid arthritis and beyond. Drug Des Devel Ther. 2014;8:349-364.

56. Rosman Z, Shoenfeld Y, Zandman-Goddard G. Biologic therapy for autoimmune diseases: an update. BMC Med. 2013;11:88. 57. Grupp SA, et al. Chimeric antigen receptor-modified T cells for acute lymphoid leukemia. N Engl J Med. 2013;368(16):1509-1518.

58. Maude SL, Barrett D, Teachey DT, Grupp SA. Managing cytokine release syndrome associated with novel T cell-engaging therapies. Cancer J. 2014;20(2):119-122.

59. Moradinejad MH, Ziaee V. The incidence of macrophage activation syndrome in children with rheumatic disorders. Minerva Pediatr. 2011;63(6):459-466.

60. Yokota S, Itoh Y, Morio T, Sumitomo N, Daimaru K, Minota S. Macrophage Activation Syndrome in Patients with Systemic Juvenile Idiopathic Arthritis under Treatment with Tocilizumab. J Rheumatol. 2015;42(4):712-722. 